\title{
La lengua española en la fonología estructuralista americana*
}

\author{
Spanish language in American structuralist phonology
}

\author{
ESTRELLA RAMÍREZ QUESADA \\ Universidad de Córdoba, Facultad de Filosofía y Letras. España. \\ 162raque@uco.es
}

Este artículo aborda el estudio historiográfico de las líneas fundamentales del estructuralismo americano o distribucionalismo en el ámbito de la fonología del español. Así, se esbozan los fundamentos de esta tendencia lingüística y se analizan los principales trabajos que aplican sus principios a la caracterización fonológica del español. Estos estudios, a veces poco conocidos, suponen tempranas reflexiones fonológicas y muestran la especificidad de la interpretación de esta escuela en comparación con los trabajos europeos. Se pretende contribuir de este modo al conocimiento de la historia de los estudios fonológicos del español.

Palabras claves: fonología, lengua española, distribucionalismo, estructuralismo americano, historiografía lingüística

This paper deals with the historiographical study of the main lines of American structuralism or distributionalism in the field of Spanish phonology. Thus, the grounds of this linguistic trend are outlined, and the main works applying its principles to the phonological characterization of Spanish are analyzed. These studies, little known sometimes, represent early phonological reflections and show the specific interpretation of this school compared to European works. Consequently, this paper aims to contribute to the knowledge of the history of phonological studies of Spanish.

Key words: phonology, Spanish language, distributionalism, American structuralism, linguistic historiography

\footnotetext{
Quiero expresar mi agradecimiento al Dr. Francisco Javier Perea Siller (Universidad de Córdoba, España), por los comentarios a la primera versión de este artículo, así como a los evaluadores del texto, por sus sugerencias durante el proceso de revisión.
} 
En las siguientes páginas trataremos de dar una visión de conjunto de los principales comentarios que la lingüística estructural americana -desde los ańos treinta hasta la aparición del generativismo- ha aportado sobre la fonología del español. Para ello, introduciremos las nociones fundamentales del distribucionalismo norteamericano, con especial atención a la fonología, antes de esbozar un panorama de las ideas en torno al vocalismo, el consonantismo y los suprasegmentos del español ${ }^{1}$. Pretendemos contribuir así al conocimiento historiográfico tanto de la lingüística americana de la época como de las visiones de la fonología del español de este período, tradicionalmente eclipsadas o, en cualquier caso, bastante desconocidas, con la excepción del estudio de los elementos suprasegmentales, que estimamos suficientemente documentado gracias, entre otros, a los capítulos de Martínez Celdrán (2003) y Dorta (2007)².

\section{El estructuralismo lingǘístico en Norteamérica}

De acuerdo con Lepschy (1971 [1966]: 98 y ss.), la lingüística norteamericana había comenzado a mostrar su especificidad frente a la tendencia europea a partir de los ańos veinte, sobre todo con los estudios de Sapir. No obstante, Robins (1987 [1976]: 207) recuerda que tres de las figuras principales, Boas, Sapir y Bloomfield, no permanecieron ajenas a los estudios europeos y apunta que las décadas de más influencia de la lingüística de Bloomfield-aunque en algunos casos no se siguieran sus principios de manera estricta- son los años treinta y cuarenta, de manera que este período, junto con la década de 1950, es conocido como "era bloomfieldiana". Sobre la denominación de esta época, Hymes y Fought (1981: 116) indican que son diversos los nombres (post-Bloomfieldian, neo-Bloomfieldian, Bloomfieldian), mientras que Anderson (1990: 313) prefiere hablar de estructuralismo norteamericano. Otra opción frecuente es la etiqueta descriptivismo. En nuestro caso, empleamos también la denominación distribucionalismo para referirnos a esta escuela lingüística de manera general.

Las líneas fundamentales de la lingüística estructuralista norteamericana son el interés por la sincronía y el estudio de la estructura sintagmática, con especial atención a la distribución y el análisis en constituyentes inmediatos. El método de trabajo, de carácter inductivo, adquirió gran relevancia, y dejar constancia de la manera más explícita posible del procedimiento de análisis se convirtió en uno de los objetivos fundamentales de los estudios. Además del interés por la enseñanza de lenguas y el rigor metodológico, que conforman dos de las señas de identidad del estructuralismo norteamericano, es importante destacar en este sentido la conciencia de la lingüística como ciencia independiente. En 1924, la fundación de

\footnotetext{
${ }^{1}$ Para unificar la presentación del estudio, empleamos en las transcripciones la última versión del AFI (2015), si bien en algunos casos, dado su interés, hacemos referencia, indicándolo, al símbolo original utilizado por sus autores.

${ }^{2}$ También Quilis (1975), desde un punto de vista más general y no tan centrado en los estudios del español, había dedicado algunos comentarios a la entonación según los principios del estructuralismo norteamericano.
} 
la Sociedad Lingüística de América y la edición de la revista Language ejemplifican el auge de la lingüística como disciplina dotada de autonomía y de un método científico. Dicho método, como es sabido, se asoció rápidamente con el distribucionalismo; así, el análisis en función de la distribución fue visto como un medio para estudiar los elementos lingüísticos atendiendo únicamente a criterios estrictamente sistemáticos, como el contexto de aparición.

Hymes \& Fought (1981: 116-117) señalan que el estructuralismo americano ha sido objeto de poca atención historiográfica, y atribuyen a la crítica llevada a cabo por Chomsky y sus seguidores el hecho de que los trabajos distribucionalistas hayan quedado ensombrecidos. Además, son tópicas algunas de las afirmaciones que, a su juicio, se han vertido sobre este período: "In historiography of American structuralism two explanatory themes have been introduced so often as to amount to clichés: behaviorism and antagonism to meaning, on the one hand, and anthropological field work, on the other" (Hymes \& Fought 1981: 6). Pero lo cierto es que Bloomfield no desechó el significado en su explicación lingüística, aunque su concepción mecanicista y conductista, frente al mentalismo de Sapir, le hicieron centrarse en la metodología y en el análisis formal (Robins 1987 [1976]: 206), puesto que, a diferencia del significado, eran elementos sistematizables. El aspecto ante el que Bloomfield se mostraba contrario era el psicologismo, no la semántica, a la que, con todo, reconocía dificultades de estudio (vid. Matthews 1993). No obstante, algunos de sus seguidores, entre los que se sitúan Bloch y Trager, trataron de eliminar por completo el recurso a la semántica de sus descripciones y optaron por una separación entre el nivel fónico y el gramatical. Pero no se trató de una opción extendida a todos los autores ni exenta de críticas, incluso dentro de la propia corriente (vid. Pike 1947).

\section{LOS PRINCIPIOS FONOLÓGICOS DE LA ESCUELA DISTRIBUCIONALISTA}

La perspectiva adoptada por la mayoría de los estructuralistas norteamericanos para definir el fonema guarda relación con la de autores ingleses como Daniel Jones (Martinet 1973 [1968]: 75). Como el propio Jones (1973 [1957]: 27) declara, comparte con Baudouin de Courtenay la idea de que el fonema es de naturaleza fonética, lo cual supone una diferencia con respecto a los lingüistas del Círculo de Praga. La teoría del fonema entendido como elementos significativos del habla estaba ya establecida en Inglaterra en torno a los años veinte, de manera que, en virtud de esta caracterización, Jones (1973 [1957]: 2428) sitúa la fonología como parte esencial de la fonética. La fonología, vista desde este ángulo, se ocupa de un determinado tipo de sonidos producidos en el habla -aquellos que conllevan diferencias significativas- y tiene naturaleza fonética. Para Bloomfield, "the study of significant speech-sounds is phonology or practical phonetics. Phonology involves the consideration of meanings" (1984 [1933]: 78) ${ }^{3}$.

\footnotetext{
${ }^{3}$ Mantenemos la cursiva del original.
} 
A partir de los principios fundamentales de Language, y motivados por las discusiones que se extendían por las principales escuelas fonológicas en este período, los autores norteamericanos desarrollaron un debate en torno a estos conceptos ${ }^{4}$. Como muestra, debemos aludir a Swadesh, quien en "The Phonemic Principle" (1934) señala:

The phonemic principle is that there are in each language a limited number of elemental types of speech sounds, called phonemes, peculiar to that language; that all sounds produced in the employment of the given language are referable to its set of phonemes; that only its own phonemes are at all significant in the given language (Swadesh 1934: 118).

A partir de la relación entre los sonidos y los fonemas se llegará a una de las premisas del análisis fonológico distribucionalista: la búsqueda de la biunicidad o biunivocidad (biuniqueness). De acuerdo con este principio, al que Harris dio nombre (Anderson 1990: 335), es posible trazar la representación fonética de un enunciado a partir de una representación fonológica, y viceversa: a partir de una representación fonética, establecer su representación fonológica, cuestión esta última no siempre aceptada dentro de la corriente (Lepschy 1971 [1966]: 166-171). La especificación de la distribución de cada alófono permitía explicar la realización fonética de cada fonema en las distintas posiciones. La Escuela de Praga, en cambio, contaba con otras nociones, como las de variantes fonéticas facultativas o las de neutralización y archifonema, para dar cuenta de las posibilidades de realización de los fonemas. En el caso de la fonología norteamericana, el recurso a la distribución complementaria, término posiblemente acuñado por Swadesh (Jones 1973 [1957]: 22), suplía la ausencia de estos conceptos, aunque los propios autores eran conscientes de que la asignación de una realización a un fonema determinado no siempre podía llevarse a cabo en virtud de este criterio.

Algunas dificultades que señala Gutiérrez Ordóńez (1983: 104) a propósito de la teoría fonológica de estos autores son determinadas variaciones libres, la distribución deficitaria y el recubrimiento (también conocido como solapamiento, denominación que emplearemos, del inglés overlapping). En el primer caso, hay variantes que responden a la libertad del hablante y no se explican, por lo tanto, en función de la distribución del fonema. No obstante, debemos señalar que, transcurridos algunos ańos, los autores fueron dando cabida a la variación libre en sus descripciones (vid. \$3). En cuanto a la distribución deficitaria, Gutiérrez Ordónez recoge el ejemplo de Bloch y Trager de los fonemas /t/ y /d/ del inglés tras el segmento /s/, contexto en el que solo aparece /t/. Debemos señalar que, con respecto a la asignación de los alófonos a los fonemas oclusivos del inglés tras $/ \mathrm{s} /$, Swadesh (1934: 124) había señalado que la variante que pudiera ser asignada a dos fonemas

\footnotetext{
${ }^{4}$ Hay que destacar que no todos los autores se mostraron de acuerdo con la noción de fonema y el carácter de la fonología que acabamos de esbozar, aunque fuera la visión más generalizada. Para tener otra perspectiva, puede consultarse Twaddell (1935).
} 
se otorgaría al fonema con el que guardara más semejanza fonética, y si ese sonido era igualmente parecido a cualquiera de los dos, se le consideraría fonémicamente independiente (phonemically independent). Pero no solía hacerse así. Wang (1973: 103-104) indica que el segundo segmento de spin comparte con el fonema /p/ que es sordo y tiene en común con el fonema /b/ que es no aspirado, por lo que se sitúa entre ambos. Sin embargo, suele asignarse al primero de ellos en virtud de la coherencia del sistema, debido a que el fonema /s/ va seguido de los fonemas sordos cuando existe un correspondiente sonoro (/sf/, pero no /sv/), aunque plantea la influencia ortográfica como posible factor. La tercera dificultad sobre la que repara Gutiérrez Ordóñez es el solapamiento, es decir, la asignación de un sonido a más de un fonema. Como el lingüista asturiano comenta, en "Phonemic overlapping" (1941) Bloch había distinguido un solapamiento parcial y un solapamiento completo. En el primer caso, un sonido podía ser asignado a un fonema u otro dependiendo del contexto en el que se encontrara. En el segundo, el mismo sonido en el mismo contexto podría ser considerado como variante de más de un fonema, lo que rompería el principio de biunicidad. Anderson apunta que a partir de ahí los autores trataron de evitar los solapamientos totales (Anderson 1990: 334-335).

También debemos indicar el interés del distribucionalismo por el estudio de los rasgos prosódicos y los fenómenos de juntura, de los que nos ocuparemos más abajo. Así, para finalizar esta breve contextualización, cabe añadir que la sistematización de los métodos y resultados alcanzados en la fonología norteamericana de la época es la llevada a cabo por Hockett en A Manual of Phonology (1955), que críticos como Lepschy (1971 [1966]: 167-168) consideran la correspondencia americana de los Principios (1939) de Trubetzkoy en Europa. No obstante, en esta obra se percibe ya la influencia de Roman Jakobson en la consideración del fonema, y resulta notorio que, a pesar de que la base siga siendo la fonología distribucionalista, Hockett aluda con mayor frecuencia que sus predecesores a Jakobson, Trubetzkoy y otros autores de la tradición europea en la exposición de sus ideas.

En suma, la amplia dedicación a los principios de la fonología y el interés que despertó el análisis fonémico, justificado por Bloch y Trager en una de las secciones de su Outline of Linguistic Analysis (1942: \$3.2), es entendible desde la perspectiva de estos autores, herederos de la tradición de unos estudios antropológicos cuyo énfasis se había situado hasta entonces en la transcripción fonética de lenguas desconocidas. La fonología se concibió, así, como estudio estructurador del amplio material fonético, por lo que se erigió en una de las muestras más importantes del auge de una lingüística dotada de autonomía, con un objeto de estudio propiamente delimitado y un rigor metodológico sin precedentes.

\section{LAS VISIONES DE LA FONOLOGÍA DEL ESPAŃOL}

Nos centraremos a continuación en algunas de las ideas planteadas en torno a la fonología del español desde el punto de vista del estructuralismo americano. Para ello, abordaremos algunos comentarios de autores como Bloomfield (1933) o Hockett (1955) 
en obras generales, esto es, no dedicadas a la lengua española. Además, trataremos estudios específicos sobre la fonología del español. Así, tenemos trabajos globales, como el de Trager (1939), quien elaboró un análisis fonológico en "The Phonemes of Castillian Spanish", a partir del cual surgieron propuestas como las de Chavarría-Aguilar (1951) y Silva-Fuenzalida (1953) para otras variedades hispánicas -costarricense y chilena, respectivamente-, a las que se suma la de King (1952), para el español de México. Estas aportaciones se sitúan en la tercera etapa, estructuralista, de las tres que delimita Erica García (1968) en relación con los estudios fónicos hispanoamericanos. Con anterioridad a esta fase, los estudios se caracterizaban por presentar las particularidades fonéticas de los distintos dialectos, pero, a partir de entonces, dos son los rasgos fundamentales: "in the first place, American Spanish is no longer studied within the framework of general Spanish dialectal developments; second, Latin American scholars began to be influenced by foreign, i.e. non Hispanic, schools of linguistics" (García 1968: 70). Y en efecto, la influencia fundamental de los autores que analizaron fonológicamente la lengua española en los inicios de los años cincuenta fue la de los lingüistas norteamericanos y sus principios, en gran parte de los casos en universidades estadounidenses o en estrecha colaboración con lingüistas de tales instituciones. La excepción se sitúa en la zona del Río de la Plata, en la que se observa la presencia de los principios de la Escuela de Praga (García 1968: 71). Asimismo, debemos señalar que, más allá de las caracterizaciones de conjunto, también tendremos en cuenta otros estudios que tratan cuestiones puntuales, como las semivocales, el acento o los fenómenos de juntura ${ }^{5}$.

\subsection{El vocalismo}

El inventario del español es aducido a menudo como ejemplo de sistema de cinco vocales. Así ocurre en Bloomfield (1984 [1933]: 105), autor que sitúa las lenguas con este sistema pentavocálico entre las más sencillas atendiendo a este criterio, aunque indica que existen sistemas aún más reducidos de tres vocales. En cualquier caso, el bajo número de fonemas vocálicos de lenguas como el español es causante de un alto grado de variación; de este modo, a juicio de Bloomfield, en español las vocales medias oscilan bastante entre las posiciones más altas y las más bajas (con cualidades similares a las de las italianas, en las que conforman fonemas distintos). El esquema empleado por Bloomfield es similar al que utilizará el resto de autores distribucionalistas, pues remite al clásico triángulo vocálico de Hellwag, empleado en los estudios de fonética. Así, el esquema denominado por Hockett $2+2+1$ es uno de los más frecuentes. A diferencia de Trager, que, aun considerándolo importante, señala el carácter añadido del redondeamiento labial (Trager 1939: 217), Chavarría-Aguilar indica que la posición de la lengua y la labialización actúan en español a la vez, sin que se aprecie predominio de un rasgo sobre otro: "There is contrast ${ }^{6}$ in both

\footnotetext{
${ }^{5}$ También siguen de manera general estos principios los estudios de fonología contrastiva inglés-español, como los de Bowen (1956), Lado (1956), Cárdenas (1960) y Stockwell y Bowen (1965).

${ }^{6}$ En la fonología americana, el término contraste hace referencia al intercambio de segmentos que ocasiona
} 
tongue position and labialization between the members of the two pairs: the front vowels have the lips neutral, the back vowels have the lips rounded" (Chavarría-Aguilar 1951: 248). En la misma línea se sitúa Hockett (Hockett 1955: 85).

Con respecto a las variantes -término que fue dejando paso a alófono-, Trager (1939: 217) sigue los datos fonéticos de Navarro Tomás (1932) para seńalar la existencia de vocales abiertas, pero, a pesar de que Navarro Tomás da contextos de nasalización, no considera variantes nasalizadas. Sí lo hace Silva-Fuenzalida para el español de Chile, quien introduce la variación libre en este caso: "La nasalización de las vocales es un elemento no-distintivo, ya que varía libremente con cero u ocurre siempre en la vecindad de una consonante nasal. Está, además, en variación libre con [n] antes de /x/" (Silva-Fuenzalida 1953: 151) y pone como ejemplos [na' rajxa] y [na' rãxa], 'naranja'.

De los autores estudiados, King es el menos cercano a Trager. A modo de ejemplo, no considera contextual la abertura vocálica del alófono [e], sino que esta articulación incluye la posibilidad de presentar fonos de distinta abertura (King 1952: 52). En cambio, en la distribución de las variantes abiertas y cerradas de las vocales, Silva-Fuenzalida se muestra muy próximo a Trager (Silva-Fuenzalida 1953: 156). Constatamos así una esencial diferencia entre la descripción de Trager y las posteriores: mientras que en el primer caso no tenía cabida ninguna realización que no dependiera del contexto, el ámbito de la variación libre se suma al de la distribución complementaria y se hace presente en los estudios posteriores. Como causas podemos apuntar la evolución del estructuralismo americano así como el hecho de que, aunque trabajen en colaboración con autores distribucionalistas y bajo los principios fonológicos de esta escuela, algunos de estos lingüistas son hablantes de español y conocen la tradición de estudios hispánicos en dialectología y fonética, lo que sin duda supone una gran influencia en la perspectiva que adoptan, menos estricta en el plano estructural y más consciente de la variación, a la que tratan de dar cabida y respuesta desde el punto de vista fonológico.

En el caso de agrupamientos de vocales, Bloomfield (1984 [1933]: 125) opina que, en español, como en italiano, las combinaciones de los elementos que transcribe [je, we] son bifonemáticas, a diferencia de otras lenguas, en las que tienden a ser tratadas estructuralmente como fonemas únicos. Trager (1939) también considera la existencia de variantes no silábicas, aunque hay secuencias de dos vocales silábicas. No es el caso de Chavarría-Aguilar, quien no cree que existan en el español costarricense secuencias como / oe/, /eo/ y /ea/, y afirma que la primera es no silábica (Chavarría-Aguilar 1951: 249). Así, son fonéticamente idénticas las realizaciones de las vocales de la primera sílaba en puerta y poesia (/ue/), fonéticamente [we]. Tampoco se da la secuencia/ea/, de manera que tenemos en su lugar/ia/ (/pelia'ria/ en pelearía), y ocurre lo mismo en /eo/ // pior/ en el caso de peor).

distinciones significativas, a diferencia de muchos autores de la tradición europea, que prefieren hablar de oposición en estos casos. De esta manera, en lugar de contrastar, en la fonología europea se opta por conmutar, y la operación de intercambio de unidades para determinar las distinciones significativas es la conmutación. El concepto de contraste se sitúa así en el plano sintagmático y el de oposición se reserva para el paradigmático. Consúltese a este respecto el prefacio de Martinet (1974 [1955]). 
También Bowen (1956-1957) se ocupa de la realización de las distintas secuencias vocálicas. Entre sus informaciones, que interesan más desde el ámbito de la fonética, destaca el apoyo a la idea de juntura, que considera "bien establecida" entre el inventario de fonemas del español (Bowen 1956-1957: 5). En el artículo, señala los distintos patrones de realización de las vocales en contacto; por ejemplo, cuando aparecen dos vocales iguales seguidas, tiende a pronunciarse una única. Por otro lado, cuando las vocales son distintas, la primera de ellas suele verse modificada. Así, los fonemas /i/ y /e/ seguidos de determinadas vocales pasan a ser el fonema semivocálico que transcribe /y/ (1956- Bowen 1957: 9). Ocurre lo mismo con los fonemas /u/ y /o/, que en determinadas ocasiones cambian a /w/. Cuando la secuencia es /ae/, /ao/, /ei/, /oi/, /ou/, puede llegar a desaparecer el primer fonema (Bowen 1956-1957: 11-12). En cualquier caso, las realizaciones varían en función de la acentuación de las vocales.

La denominación de semivocales es la empleada generalmente en la bibliografía americana tanto en el caso de las unidades que tradicionalmente se conocían como semiconsonantes -el primer elemento del diptongo creciente- como en el de las semivocales -el segundo elemento del diptongo decreciente-, en aras de una mayor simplificación. Además de en la denominación, la consideración de estas unidades se vio influida por su importancia en los análisis de la lengua inglesa, que fueron en varios casos la referencia. King planteó la existencia de los fonemas que transcribe /w/ y /y/, semivocales, en el cuadro de consonantes (King 1952: 51-52). La visión que en este sentido ha generado mayores repercusiones es la de Bowen \& Stockwell (1955), quienes opinan que las semivocales no son alófonos de los segmentos /i/ y /u/, sino de los fonemas que transcriben /y/ y /w/. En su interpretación, cada uno de ellos cuenta con un alófono espirante y otro no espirante (es decir, consonántico y semivocálico). A juicio de estos autores, uno de los criterios fundamentales a la hora de realizar esta asignación es la mayor simplicidad que quedaría en la descripción. Así, en el caso del morfema verbal de tercera persona, tendríamos el mismo fonema, que transcriben $/ \mathrm{y} /$, en comió-leyó (/ko'myo/, /le'yo/) y comieron-leyeron (/ko'myeron/, /le'yeron/), con distinta realización alofónica ${ }^{7}$. Al incluir en estos fonemas alófonos espirantes, Bowen y Stockwell se ven obligados a recurrir a la juntura para distinguir las secuencias hay una de ayuna, que transcriben mediante los mismos fonemas: /ay+ 'una/

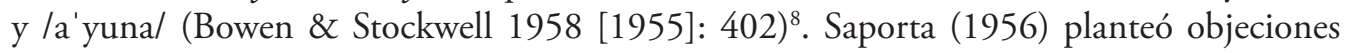
a estas ideas, a lo que siguió una respuesta, reafirmándose en su posición, de Bowen \& Stockwell (1956). Poco después, Saporta \& Cohen hicieron la siguiente afirmación: "the two non-syllabic phonemes /i/ and /u/ are referred to as semivowels. These, incidentally, are

\footnotetext{
${ }^{7}$ Alarcos no ve necesariamente una simplificación en este caso, e incorpora la respuesta a estos autores a partir de la tercera edición de la Fonología española (Alarcos 1959: 185).

${ }^{8}$ Los autores no indican dos acentos en la secuencia hay una. La posición del acento en hay una y ayuna se representa de igual manera: sobre la vocal $u$; en nuestro caso, hemos seńalado el acento según el AFI, precediendo la sílaba acentuada de la palabra correspondiente, lo que comporta una diferencia en la representación fonológica. La presencia de dos acentos en hay una, y no solo la juntura, sería otra diferenciación entre las dos secuencias.
} 
to be further distinguished from the consonantal phonemes /y/ and /w/" (Saporta \& Cohen 1958: 372). En este caso, son tratados como una categoría aparte, pero sin llegar a integrarse entre los fonemas consonánticos. Esto supone la prueba de que se trata de una cuestión que no ofrece una respuesta única.

\subsection{El consonantismo}

En la descripción de los diferentes sistemas de obstruyentes, Hockett hace mención de la lengua española, que clasifica junto a lenguas como el alemán y el takitaki entre los sistemas que cuentan con dos series de oclusivas (/p, t, k/ y /b, d, g/) y una de fricativas (/f, s, x/) (Hockett 1955: 112-113). No obstante, señala que el español presenta algunas peculiaridades, puesto que cuenta con fonemas africados o la posibilidad de que aparezca el fonema $/ \theta /$. No encontramos el fonema $/ \theta /$ ni el fonema $/ K /$ en las variedades hispanoamericanas. Por ejemplo, Chavarría-Aguilar había indicado la existencia de dieciocho fonemas consonánticos en el español de Costa Rica (Chavarría-Aguilar 1951: 248). Para King son veinte, pues incluye en su caracterización de la variedad mexicana los fonemas /w/, /y/ y / / /, además de los habituales (King 1952: 52). Iremos, pues, comentando las distintas series, a excepción de los fonemas líquidos (laterales y vibrantes), puesto que no ofrecen en su caracterización diferencias notables entre sí ni con respecto a los estudios realizados desde otras perspectivas ${ }^{9}$. En muchos casos, las divergencias entre unas y otras propuestas suelen ser de índole fonética, dada la variabilidad de los dialectos hispánicos.

En primer lugar, destaca la consideración de la existencia de variantes espirantes fricativas en el caso de los fonemas "oclusivos" -que Trager escribe entre comillas precisamente por ser una denominación inexacta (Trager 1939: 218)-. Señala este autor que los fonemas oclusivos sonoros poseen realizaciones no oclusivas -denominadas espirantes no fricativas, para distinguirlas de las fricativas- en todos los contextos excepto en posición inicial absoluta y tras nasal (en el caso del fonema /d/, también tras /l/, siguiendo la caracterización fonética de Navarro Tomás [1932]).

La distinción de los autores americanos entre espirantes fricativas y no fricativas se ha visto corroborada con el tiempo, a través de diferentes denominaciones (la más extendida en los estudios hispánicos, la de fricativas y aproximantes, desde Martínez Celdrán [1984]). Así, recientemente, Martínez Celdrán, siguiendo la propuesta de Martinet (1956), ha recuperado la etiqueta espirante, que en otros casos se usaba como sinónimo de fricativo, y ha denominado de este modo la subclase de aproximantes centrales no róticas entre las que se incluyen los sonidos [ $\beta_{\tau} \partial_{\tau}$ ช $]$ del español (Martínez Celdrán 2004: 204-205).

Con respecto a los fonemas oclusivos en posición implosiva, Trager opina que la realización será sonora o sorda en función de la consonante que siga, y se asignará al fonema sordo o sonoro correspondiente (Trager 1939: 218, 221). No emplea el concepto de

\footnotetext{
${ }^{9}$ Acaso podemos citar la mención del fonema vibrante múltiple como secuencia de dos fonemas en Stockwell, Bowen \& Silva-Fuenzalida (1958 [1956]: 407).
} 
neutralización, ajeno al distribucionalismo, de manera que opta por el criterio sintagmático a la hora de consignar las variantes. Chavarría-Aguilar comparte la visión de Trager en la asignación de alófonos espirantes en posición implosiva a los fonemas sordos o sonoros (Chavarría-Aguilar 1951: 249-250). También Silva-Fuenzalida sigue los principios de Trager, aunque menciona un cierto grado de variación libre y seńala que los alófonos no oclusivos de los fonemas /b, d, g/ son fricativos (Silva-Fuenzalida 1953: 159-160). King se separa algo de esta línea, pues no documenta los fonemas sordos en posición implosiva en interior de palabra, de tal forma que las variantes sonoras y ensordecidas se asignan a los fonemas /b, d/, ya que no considera el fonema /g/ en posición implosiva (King 1952: 54).

Hockett, por su parte, indica la presencia de variantes espirantes para los fonemas /b, d, g, dj̄ y su posible ensordecimiento en determinados contextos (Hockett 1955: 113). La posibilidad de articulaciones bilabiales espirantes en español también queda recogida en Bloomfield (1984 [1933]: 101). La denominación de espirantes, como puede observarse, es preferida por la escuela norteamericana.

En el caso de los fonemas africados, se plantea la cuestión de analizarlos como uno o dos fonemas $(/ \mathrm{t} /+/ \mathrm{J} /, / \mathrm{d} /+/ \mathrm{j} /)$. Bloch $\&$ Trager los consideran dos fonemas en el caso de la lengua inglesa (Bloch \& Trager 1942: 49), pero, en español, Trager interpreta un único fonema (Trager 1939: 219). El motivo parece ser una búsqueda de simetría en la descripción, puesto que, por ejemplo, en español habría que añadir un fonema / $/$ que no aparece en otro contexto que no sea tras / $/ \mathrm{t}$. Para mostrar las oscilaciones que se plantean en torno a esta idea, hay que señalar que, años después, Trager \& Smith consideran que también en inglés se trata de un único fonema (Trager \& Smith 1965 [1951]: 34).

Por su parte, los fonemas fricativos -o espirantes fricativos, más específicamente-se subdividen en dos categorías para los autores que siguen el estructuralismo norteamericano. Los fonemas /f, $\theta, \mathrm{x} /$ son considerados espirantes alargados (slit spirants), mientras que el fonema $/ s /$ es espirante redondeado (rill spirant). Esta distinción permite separar los fonemas $/ s /$ y $/ \theta /$, a veces problemáticos en su clasificación en otras descripciones del español; recuérdese a este respecto la asignación del fonema /s/ al orden palatal llevada a cabo por Alarcos (1950: \$108, \$\$110-113). La división de las consonantes fricativas en dos grupos es común a los principales autores del distribucionalismo, y proviene de la fonética tradicional. En los autores hispanoamericanos no aparece, en parte porque la inexistencia del fonema $/ \theta /$ permite mayor facilidad en la asignación de rasgos en función del lugar de articulación.

En su interpretación fonológica, estos fonemas cuentan con realizaciones sonoras ante otra consonante sonora, por lo que la sordez no es distintiva. Ya Trager había señalado que la correlación de sonoridad solo está presente en los fonemas oclusivos y los africados (Trager 1939: 218). En otras variedades, la caracterización es diferente, ya que no aparece el fonema $/ \theta /$ y sí un fonema /h/. Por ejemplo, en el caso de Chavarría-Aguilar, el fonema $/ \mathrm{h} /$ ocupa el lugar del fonema velar ${ }^{10}$, pero para Silva-Fuenzalida ambos están presentes

${ }^{10}$ La realización del fonema/s/ en distensión silábica en el español costarricense es [s], por lo que no se produce 
(Silva-Fuenzalida 1953: 159); el fonema /h/ se manifiesta en formas como [loha' miyoh] los amigos. Sin embargo, en otros lugares no se considera necesario dar estatus de fonema a este elemento, como veremos (\$3.3). Para King, de hecho, es una posibilidad articulatoria del alófono [s], que describe del siguiente modo: "[s], varying from a lax [s] to [h], occurring in free variation with [s] before voiceless consonants and before pause" (King 1952: 56) ${ }^{11}$.

Finalmente, es interesante señalar la consideración de los fonemas nasales. Los tres fonemas que entran dentro de esta categoría son $/ \mathrm{m} /, / \mathrm{n} / \mathrm{y} / \mathrm{n} /{ }^{12}$. El primero de ellos aparece en posición implosiva ante consonante labial (a lo que King [1952: 55] añade el final absoluto: álbum) y posee una variante labiodental ante /f/; así, Trager adscribe los alófonos $[\mathrm{m}]$ y $[\mathrm{m}]$ al fonema $/ \mathrm{m} /$ por similitud fonética ${ }^{13}$. Por otro lado, en posición final absoluta solo es posible $/ \mathrm{n} /$, pero se produce un cambio morfofonológico de $/ \mathrm{n} / \mathrm{a} / \mathrm{m} /$ cuando en posición final de palabra encontramos $/ \mathrm{n} /$ y la siguiente palabra empieza por un fonema labial (/p, b, f, m/) (Trager 1939: 222). Esta solución difiere, de nuevo, de la opción de la neutralización de los fonemas nasales en posición implosiva que plantean la fonología de la Escuela de Praga y sus seguidores. A continuación, veremos otra interpretación de este cambio de fonemas en relación con la noción de juntura.

\subsection{Los fenómenos de juntura}

Los efectos de las transiciones han sido objeto de estudio por parte de los autores distribucionalistas. Así, referiremos brevemente algunas de las ideas sobre estos fenómenos, para lo cual tendremos en cuenta las principales propuestas en el ámbito del español: Kahane y Beym (1948), Silva-Fuenzalida (1951) y Stockwell, Bowen \& Silva-Fuenzalida (1956) ${ }^{14}$.

Kahane \& Beym, a partir de la observación del español de México, señalan casos de juntura cerrada entre palabras -esto es, en los que no hay transición especial, sino unión entre una y otra- a tenor de la variación alofónica (Kahane $\&$ Beym 1948). Así, hay juntura cerrada cuando una palabra finaliza en $[\mathrm{m}],[\mathrm{y}]$ o $[\mathrm{z}]$ (ya que estos sonidos no se producen ante pausa); ponen el ejemplo de acoges mis tonterías, en el que el sonido final del verbo viene condicionado por el inicio del determinante posesivo. De modo similar, se produce juntura

un solapamiento parcial entre el alófono [h] procedente del fonema /h/ con un posible alófono [h] en posición implosiva.

${ }^{11}$ King (1952: 51) estima que un grupo de fonos con aproximadamente las mismas cualidades constituye un alófono. De este modo, bajo el alófono [s] se agrupa un conjunto de sonidos posibles.

${ }^{12}$ Chavarría-Aguilar fonologiza en el espańol de Costa Rica la consonante /y/. Este fonema puede aparecer entre vocales (con eso [kon'eso] frente a conejo [ko'neho]) y alternar con el fonema cero ante otra nasal (1951: 251) (vid. infra).

${ }^{13}$ King y Silva-Fuenzalida siguen a Trager en este aspecto, pero Chavarría-Aguilar indica en este contexto la nasalización de la vocal precedente: [1̃ fame] infame (Chavarría-Aguilar 1951: 251).

${ }^{14}$ Remitimos a Estapà (1989) para profundizar en la juntura y su presencia en el espańol. No nos ocupamos de este y otros estudios, como Quilis (1964), por estar nuestro trabajo circunscrito a una corriente concreta. 
cerrada cuando una palabra comienza por $[\beta, \gamma, \gamma]$ y también en el caso de la conjunción $y$ si la siguiente palabra empieza por vocal. Asimismo, hay juntura cerrada cuando una palabra acaba en la secuencia vocal+consonante y la siguiente empieza por vocal, de manera que se silabean juntas consonante final y vocal inicial, y cuando una palabra acaba con el mismo sonido por el que empieza la siguiente, contexto en el que el hablante reproduce un único sonido ininterrumpido (1948: 389). Como puede observarse, el único supuesto que comporta cambio de fonemas es el de [m], lo que enlaza con la visión de Trager. El estudio de Kahane $\&$ Beym se ocupa de analizar los casos de juntura cerrada y abierta en los distintos contextos sintácticos, de manera que vinculan la juntura con el sintagma. Así, por ejemplo, observan (1948: 394) que un vocativo suele unirse al elemento precedente y separarse del siguiente.

Silva-Fuenzalida (1951) se basa en el estudio de Kahane \& Beym para aportar más datos, en su caso a partir del español de Chile. Hay, por lo tanto, diferencias: no existe el alófono [z], y [y] puede aparecer después de pausa, por ejemplo. En el caso de las sustituciones de fonemas, añade más casos, derivados en gran medida de su consideración de las semivocales como fonemas. Así, indica los siguientes cambios en los límites de palabras en grupos agente-acción: $/ \mathrm{i} />/ \mathrm{j} /$ ante vocal, $/ \mathrm{e} />/ \mathrm{j} /$ antes de $/ \mathrm{a}, \mathrm{o}, \mathrm{u} /, \mathrm{o} />/ \mathrm{w} /$ antes de $/ \mathrm{a}$, e, i/ y la pérdida de fonemas vocálicos en algunos contextos. Como ejemplo de sustitución de fonemas, frente al segmento final /o/ de compañero, señala (Silva-Fuenzalida 1951: 37) que en mi compañero arrancó tenemos la secuencia /wa/.

Por otro lado, King indica la existencia de juntura abierta en español (King 1952: 57), que permite, por ejemplo, distinguir entre /su+'abe/ y /su'abe/. Stockwell, Bowen \& Silva-Fuenzalida (1956) también consideran la presencia en español de una juntura interna abierta, /+/. Así, por ejemplo, en dialectos que presentan casos como [e'yoxo] (en ojo) y [e'noxo] (enojo), la representación fonológica, que evita añadir un fonema $/ \mathrm{y} /$, es /en+'oxo/ y /e'noxo/ ${ }^{15}$. Hemos aludido antes a la presencia de juntura en el caso de las semivocales; otro contexto es /as+' abas/ [a'haßah] (haz habas) frente a /a'sabas/ [a'saßah] (asabas) ${ }^{16}$. El fonema de juntura hace innecesario, para estos autores, postular un fonema /h/ (Stockwell, Bowen \& Silva-Fuenzalida 1958 [1956]: 407-411).

\footnotetext{
${ }_{15}$ También Hyman (1956) se ocupa de [y] como fenómeno que denota juntura. Apoyándose en un estudio experimental, concluye que se trata de un alófono del fonema /n/ en todos los casos.

${ }^{16}$ Como hemos visto con anterioridad, en el caso de haz habas, los autores indican un solo acento, que representan sobre la vocal en todos los casos (por lo que la única diferencia fonológica es la juntura). Al colocar el acento precediendo a la sílaba acentuada de la palabra, se observa la diferencia fonológica que viene apoyada por la juntura. Además, de incluir el acento en el verbo, la diferencia fonológica quedaría también marcada en este par por el número de acentos.
} 


\subsection{Los elementos suprasegmentales}

La importancia de los rasgos suprasegmentales es más que notable en la lingüística americana. Ello se deriva de la distinción que realizó Bloomfield entre fonemas primarios y fonemas secundarios, como señala Jones (1973 [1957]: 31-32). Así, el estatus de fonemas que les otorgó Bloomfield conllevó un amplio estudio por parte de sus seguidores del valor del acento, la cantidad y el tono, así como de la entonación y los fenómenos de juntura terminal, en las diferentes lenguas, y la creación de símbolos específicos para dar cuenta de estos hechos fónicos en las transcripciones. A título ilustrativo, cabe señalar que Bloch \& Trager (1942: \$3.7), en la sección “The phonemes of English”, consideran los siguientes elementos: juncture, stress, consonants, vowels e intonation, mientras que Trager \& Smith (1965 [1951]: 50) clasifican los fonemas del inglés en vowels, consonants, stresses, internal juncture, pitches y terminal junctures. En cambio, en los inicios de la fonología europea los prosodemas presentaban menor importancia, ya que los fonemas eran únicamente los elementos segmentales.

En el caso de la lengua española, los autores coinciden en señalar el carácter distintivo del acento. El español es citado por Bloomfield como uno de los idiomas con este tipo de acento. Además, apunta que, al igual que en las lenguas italiana y francesa, en español el acento suele indicar la silabicidad, de manera que marca, a diferencia de las lenguas germánicas, crestas de sonoridad que señalan los límites silábicos (Bloomfield 1984 [1933]: 125-126). El acento también es distintivo en español para Trager (1939: 219). Existen dos posibilidades: sílabas acentuadas frente a sílabas no acentuadas. En el último caso, puede darse un acento secundario o bien ausencia o poca elevación del tono. En este sentido, a partir de los datos de Navarro Tomás (1932 [1918]: \$173) sobre la repartición de los acentos rítmicos, Trager diseñó un esquema en el que se indica la posición del acento secundario según el número de sílabas de la palabra y el lugar en el que se sitúe su acento principal (Trager 1939: 220). King indica dos fonemas acentuales, cada uno de los cuales cuenta con dos alófonos, predecibles solo en algunos casos (King 1952: 56-57). Bolinger (1962), por su parte, señaló que el acento secundario en español no responde a reglas mecánicas: "The speaker will simply tend to put a secondary prominence on the same syllable where he would put a primary (sometimes a secondary) in some related word" (Bolinger 1962: 276). Además de la analogía entre palabras, también apuntó como factor de variabilidad su integración en la curva de entonación del enunciado. Por lo tanto, en su opinión, el acento primario es fonémico, pero no ocurre así en el caso del secundario, debido a su carácter mudable y a que no es empleado en distinciones (Bolinger 1962: 279).

De otro lado, Hockett también hace referencia al carácter del acento en español, en comparación con la lengua inglesa (Hockett 1955: 42). Ambas presentan contrastes distintivos de acento, pero en español el número y la posición de los acentos no influyen en la duración del enunciado, que vendrá determinado por el número de sílabas. En inglés, en cambio, el acento es el que marca el ritmo, de manera que dos enunciados tendrán la misma duración aproximada si tienen el mismo número de acentos, con independencia del número de sílabas 
y de cómo se distribuyan. Como se ha visto en otras ocasiones, la comparación entre lenguas se emplea con frecuencia como medio para subrayar la particularidad de los hechos fónicos.

Aunque contamos con una importante contribución en King (1952), el estudio más completo de los rasgos prosódicos del español desde un punto de vista fonológico es el de Stockwell, Bowen \& Silva-Fuenzalida (1956), quienes para los fonemas de tono, juntura y acento establecen la siguiente jerarquía en español: pitch phonemes, juncture phonemes y stress phonemes (Stockwell, Bowen \& Silva-Fuenzalida 1958 [1956]: 406). En el trabajo de estos autores podemos ver, por lo tanto, un tratamiento fonológico del tono y el acento, considerados fonemas. Con respecto a las junturas terminales, Stockwell, Bowen \& SilvaFuenzalida establecen tres fonemas en esta categoría, uno descendente, uno ascendente y otro con carácter suspensivo: / $/ /, / \uparrow$ y / / (Stockwell, Bowen \& Silva-Fuenzalida 1958 [1956]: 411-412). Asimismo, establecen tres fonemas tonales: / $/ 1$ (bajo), /2/ (medio) y /3/ (alto), cada uno de los cuales cuenta con varios alófonos (que oscilan entre el más bajo, antes de $/ \downarrow /$, y el más alto, antes de $/ \uparrow /$ ) (Stockwell, Bowen \& Silva-Fuenzalida 1958 [1956]: 413). También presentan tres fonemas en el caso del acento: /'/ (fuerte), I'/ (medio) y el acento no marcado (débil), ya que no creen que funcione el esquema de Trager. Cada uno de estos fonemas cuenta con un alófono, a excepción del acento fuerte, que posee dos variantes: fuerte [^] y más fuerte ['] (Stockwell, Bowen \& Silva-Fuenzalida 1958 [1956]: 413-414). Además, hay que destacar que plantean toda una serie de cambios en los esquemas de los fonemas suprasegmentales según el habla se produzca más rápida, llorando, en un tono más elevado, etc., lo que demuestra la amplitud de su propuesta (Stockwell, Bowen \& Silva-Fuenzalida 1958 [1956]: 416-418). En cualquier caso, para observar con mayor detenimiento estas y otras cuestiones sobre la entonación del español desde el punto de vista del estructuralismo norteamericano, remitimos, como hemos indicado al inicio, a los comentarios de Martínez Celdrán (2003) y Dorta (2007) y a las referencias bibliográficas que contienen.

\section{Conclusiones}

En síntesis, la especificidad de la fonología norteamericana, que comienza a acentuarse desde mediados de los años treinta, conlleva diferencias conceptuales y terminológicas claramente perceptibles en aspectos como la identidad del fonema, la importancia de la distribución a la hora de asignar alófonos o la consideración como fonemas de los fenómenos de juntura y los rasgos suprasegmentales, entre otros. Varias de estas nociones surgen al tratar la fonología del español desde este punto de vista, y muestran que la lengua española que fue un campo de aplicación importante de estos principios; así, se ha destacado, a raíz de la ausencia del concepto de neutralización, el recurso a la morfofonología y la juntura -en el caso de los fonemas nasales- o a las variantes condicionadas por el contexto en lo referente a los alófonos en posición implosiva de los fonemas tradicionalmente considerados oclusivos. De modo similar, es reseñable la consideración de espirantes de los alófonos no 
oclusivos de los fonemas /b, d, g/, hecho que, junto con otros aspectos, nos permite concluir que, a la luz de los autores estudiados, se nos presenta una visión bastante homogénea de la estructura fónica del español desde esta perspectiva teórica. La excepción se sitúa en las discrepancias en torno al tratamiento de las semivocales y el posible carácter fonémico de algunos elementos como $[\mathrm{h}]$ y $[\mathrm{n}]$.

También debemos destacar la apertura de las descripciones de las variedades hispanoamericanas a aspectos como la variación libre y las manifestaciones alejadas de la lengua estándar. Así, aunque hemos documentado el seguimiento del análisis de Trager, guía fundamental para la mayoría de los trabajos posteriores, los años transcurridos entre los estudios y la influencia de otras corrientes reflejan una incorporación de factores externos. De esta manera, trabajos como los de Chavarría-Aguilar y Silva-Fuenzalida no se mantienen en la ortodoxia distribucional que demuestra Trager. Con todo, debemos señalar que la huella de los autores norteamericanos es esencial en el empleo terminológico y la conceptualización teórica a la hora de delimitar los alófonos, la distribución, los fenómenos de juntura y otros aspectos relacionados. De hecho, en cuestiones como la juntura, que no habían sido tratadas por Trager (al ser una aportación de los inicios del distribucionalismo), los autores asumen los principios del estructuralismo norteamericano y dan lugar a soluciones que además de completar el análisis del español, conforman importantes contribuciones para la fonología general.

Asimismo, cabe resaltar que estos lingüistas son algunos de los primeros en tratar fonológicamente el acento y otros rasgos prosódicos en la lengua española. Por lo tanto, queremos destacar el valor histórico de los comentarios de estos autores en tanto conforman reflexiones tempranas, en algunos casos en anticipación a los estudios europeos, sobre la fonología del español.

En definitiva, a través de estas breves páginas esperamos haber contribuido al conocimiento y la puesta en relieve de la perspectiva americana en fonología, para lo cual hemos aludido a sus conceptos más destacados y a sus principales comentarios sobre la lengua española. Queda para futuras ocasiones ocuparnos del análisis de otros autores -menos centrales en la fonología americana, pero con aportaciones interesantes- y profundizar en su influencia en el desarrollo de los estudios fonológicos del español.

\section{Obras Citadas}

Alarcos Llorach, Emilio. 1959. "Semivocales y semiconsonantes espańolas". Archivum 9: 179-188. .1950. Fonología española. Madrid: Gredos.

Anderson, Stephen R. 1990. La fonología en el siglo XX. Trad. esp. Elena de Miguel Aparicio. Madrid: Visor.

Bloch, Bernard. 1941. "Phonemic Overlapping”. American Speech 16 (4): 278-284.

Bloch, Bernard \& George L. Trager. 1942. Outline of Linguistic Analysis. Baltimore: Waverly 
Press.

Bloomfield, Leonard. 1984 [1933]. Language. Chicago: University of Chicago.

Bolinger, Dwight. 1962. “Secondary Stress' in Spanish”. Romance Philology 15 (3): 273279.

Bowen, Donald. 1956. "A Comparison of the Intonation Patterns of English and Spanish". Hispania 39 (1): 30-35.

. 1956-1957. "Sequences of vowels in Spanish". Boletin del Instituto de Filología de la Universidad de Chile 9: 5-14.

Bowen, J. Donald \& Robert P. Stockwell. 1958 [1955]. "The Phonemic Interpretation of Semivowels in Spanish”. En: Martin Joos, Ed. Readings in Linguistics. Nueva York: American Council of Learned Societies. 400-402. [Orig. en Language 31 (2): 236240].

. 1958 [1956]. "A Further Note on Spanish Semivowels". En: Martin Joos. Ed. Readings in Linguistics. Nueva York: American Council of Learned Societies. 405. [Orig. en Language 32 (2): 290-292].

Cárdenas, Daniel N. 1960. Introducción a una comparación fonológica del español y del inglés. Washington D.C.: Center for Applied Linguistics of The Modern Language Association of America.

Chavarría-Aguilar, Óscar. 1951. “The Phonemes of Costa Rican Spanish”. Language 27 (3): 248-253.

Dorta, Josefa. 2007. "La entonación hispánica y su desarrollo”. En: Josefa Dorta, Cristóbal Corrales \& Dolores Corbella. Eds. Historiografía de la lingüistica en el ámbito hispánico. Fundamentos epistemológicos y metodológicos. Madrid: Arco/Libros. 161-199.

Estapà Argemí, Roser. 1989. "La juntura interna abierta en español”. Verba 16: 97-116.

García, Erica. 1968. "Hispanic Phonology". En: Thomas A. Sebeok. Ed. Current Trends in Linguistics, Vol. IV, Ibero-American and Caribbean Linguistics. La Haya; París: Mouton. 61-83.

Gutiérrez Ordóñez, Salvador. 1983. "La fonología”. En: Francisco Abad Nebot \& Antonio García Berrio. Coords. Introducción a la lingüistica. Madrid: Alhambra. 75-114.

Hockett, Charles F. 1955. A Manual of Phonology. Baltimore: Waverly Press.

Hyman, Ruth. 1956. "[y] as an Allophone Denoting Open Juncture in Several Spanish-American Dialects". Hispania 39 (3): 293-299.

Hymes, Dell H. \& John G. Fought (1981). American Structuralism. La Haya: Mouton.

Jones, Daniel. 1973 [1957]. “The History and Meaning of the Term 'Phoneme”. En: Erik C. Fudge. Ed. Phonology. Selected Readings. Harmondsworth: Penguin. 17-34.

Kahane, Henry R. \& Richard Beym. 1948. "Syntactical Juncture in Colloquial Mexican Spanish”. Language 24 (4): 388-396.

King, Harold V. 1952. "Outline of Mexican Spanish Phonology”. Studies in Linguistics 10: 51-62.

Lado, Robert. 1956. "A Comparison of the Sound Systems of English and Spanish". Hispania 39 (1): 26-29. 
Lepschy, Giulio C. 1971 [1966]. La lingüistica estructural. Trad. esp. Carlos Manzano. Barcelona: Anagrama.

Martinet, André. 1956. La description phonologique, avec application au parler francoprovençal d'Hauteville (Savoie). Ginebra: Librairie Droz.

. 1973 [1968]. "Neutralization". En : Erik C. Fudge. Ed. Phonology. Selected Readings. Harmondsworth: Penguin. 74-80. [Selección trad. del orig. en La Linguistique 1: $1-20]$.

. 1955. Economie des changements phonétiques. Traité de phonologie diachronique. Berna: Editions A. Francke S.A. Trad. esp. Economía de los cambios fonéticos. Tratado de fonología diacrónica. 2a ed. Madrid: Gredos, 1974.

Martínez Celdrán, Eugenio. 2003. "Análisis por niveles: la escuela americana”. En: Pilar Prieto. Ed. Teorías de la entonación. Barcelona: Ariel. 63-95. . 2004. "Problems in the classification of approximants". Journal of the International Phonetic Association 34 (2): 201-210.

Matthews, Peter H. 1993. Grammatical Theory in the United States from Bloomfield to Chomsky. Cambridge: Cambridge University Press.

Navarro Tomás, Tomás. 1932 [1918]. Manual de pronunciación española. 4a ed., Madrid: Centro de Estudios Históricos.

Pike, Kenneth. 1947. "Grammatical Prerequisites to Phonemic Analysis". WORD 3 (3): 155-172.

Quilis, Antonio. 1964. "La juntura en español: un problema de fonología”. En: Presente y futuro de la lengua española, Vol. II. Madrid: Cultura Hispánica. 163-171.

1975. "Las unidades de entonación”. Revista española de lingüistica 5 (2): 261-280.

Robins, Robert H. 1987 [1976]. Breve historia de la lingüistica. Trad. esp. Enrique Alcaraz Varó. Madrid: Paraninfo.

Saporta, Sol. 1958 [1956]. “A Note on Spanish Semivowels”. En: Martin Joos. Ed. Readings in Linguistics. Nueva York: American Council of Learned Societies. 403-404. [Orig. en Language 32 (2): 287-290].

Saporta, Sol \& Rita Cohen. 1958. "The Distribution and Relative Frequency of Spanish Diphthongs". Romance Philology 11: 371-377.

Silva-Fuenzalida, Ismael. 1951. "Syntactical Juncture in Colloquial Chilean Spanish: The Actor-Action Phrase". Language 27 (1): 34-37. . 1953. "Estudio fonológico del español de Chile". Boletín del Instituto de Filología de la Universidad de Chile 7: 153-176.

Stockwell, Robert P. \& J. Donald Bowen. 1965. The Sounds of English and Spanish. Chicago: The Univertisity of Chicago Press.

Stockwell, Robert P., Donald Bowen \& Ismael Silva-Fuenzalida. 1958 [1956]. "Spanish Juncture and Intonation”. En Martin Joos, Ed., Readings in Linguistics. Nueva York: American Council of Learned Societies. 406-418. [Orig. en Language 32 (4): 641665].

Swadesh, Morris. 1934. “The Phonemic Principle”. Language 10 (2): 117-129. 
Trager, George L. 1939. "The Phonemes of Castillian Spanish". Travaux du Cercle Linguistique de Prague 8: 217-222.

Trager, George L. \& Henry L. Smith. 1965 [1951]. An Outline of English Structure. 6a ed., Washington: American Council of Learned Societies.

Trubetzkoy, Nikolái S. 1973 [1939]. Principios de fonología. Trad. esp. Delia Garcia Giordano con la colaboración de Luis J. Prieto. Madrid: Cincel.

Twaddell, W. Freeman. 1935. "On Defining the Phoneme". Language 16: 5-62.

Wang, William. 1973. "Approaches to Phonology". En: Thomas A. Sebeok. Ed. Current Trends in Linguistics, Vol. X, Linguistics in North America. La Haya; París: Mouton. 101-121. 